\title{
Measurements of the X-ray Transmission Characteristics of Moxtek's AP3 $\mathrm{X}$-Ray Windows
}

\author{
Richard T. Creighton, Steven Liddiard, Sterling Cornaby \\ Moxtek Inc, Orem USA
}

Moxtek is a leader in X-ray windows for light element XRF and EDAX. Our windows are found on most EDAX detector systems throughout the world. This ultra-thin polymer membrane allows for detection of X-ray fluorescence of light elements such as boron, carbon, nitrogen, oxygen as well as heavier elements. The windows can withstand up to $1.5 \mathrm{~atm}$ of pressure without breaking. Moxtek's AP3 x-ray windows are specifically made to transmit low energy xrays, from about $50 \mathrm{eV}$ and above $[1,2]$.

We have implemented a system for measuring X-ray transmission of our windows at Moxtek using a JEOL SEM 6010LA with JEOL detector and software. We have constructed a test fixture inside the SEM's sample chamber which allows the placement of X-ray window films directly in front of the EDAX detector. The electron beam from the SEM hits a uniform, specially prepared sample containing $\mathrm{B}, \mathrm{C}, \mathrm{N}$, and $\mathrm{O}$ which produces characteristic X-rays from each of these elements. The X-ray transmission of an X-ray window film, such as Moxtek's AP3window films, is evaluated by taking an EDAX measurement with the film in front of the detector and then a second EDAX measurement without the window in front of the detector. This gives a measured value for the X-ray transmission of the window for these four soft X-ray producing elements.

The measured X-ray transmission from the four elements, along with knowing the film's multilayer structure, allows us to fit an X-ray transmission curve to the measured data. This Xray transmission fit adjusts the film thicknesses to match the measured X-ray transmission using a non-linear Levenberg-Marquardt fitting algorithm. From this fitting we get both the X-ray transmission curve and the thicknesses of the films which make up the X-ray window.

Also, we have measured the X-ray transmission of the AP3 films in $1 \mathrm{eV}$ resolution from 100$1300 \mathrm{eV}$ at the Advanced Light Source (ALS) at Lawrence Berkeley National Laboratory. This full spectrum measurement gives the near edge absorption transmission values of the AP3 windows, which are discounted in transmission models. Other similar transmission studies on these films have been done previously [1,2]. In this paper, we add the differences between the expected transmissions, based on the Moxtek window design, to the measured transmission. The measured x-ray transmission curves are directly compared to modeled transmission curves from tabulated $\mathrm{x}$-ray absorption values.

The transmission of two AP3.3 films and two AP3.7 films was measured on beamline 6.3.2 from $108 \mathrm{eV}$ to $1300 \mathrm{eV}$ (Figure 1). The beamline was able to cover this range of soft x-ray energies using a number of filter and grading configurations on the beamline. The measured and modeled transmission curves matched reasonably well away from absorption edges. The transmission models are quite different from the measured transmission near the absorption edges, as expected. The near-edge transmission is important in some applications [2]. The tested AP3 films were manufactured together; therefore differences between the films will show variation 
within a single manufactured batch. The films within a single manufactured batch have virtually identical transmission characteristics.

References:

[1] M. Williams, E. Hansen, A. Reyes-Mena, and D. Allred, The Transmittance of Thin Polymer Films and their Suitability as a Support Substrate for a Soft X-ray Telescope, EUV, X-Ray, and Gamma-Ray Instrumentation for Astronomy II, Oswald H. Siegmund and Richard E. Rothschild, Editors, Proc. SPIE 1549, 147-154 (1991). SO

[2] Scholze, F., Procop, M. Detection efficiency of energy-dispersive detectors with low-energy windows X-Ray Spectrometry, 34 (6), 473-476 (2005)

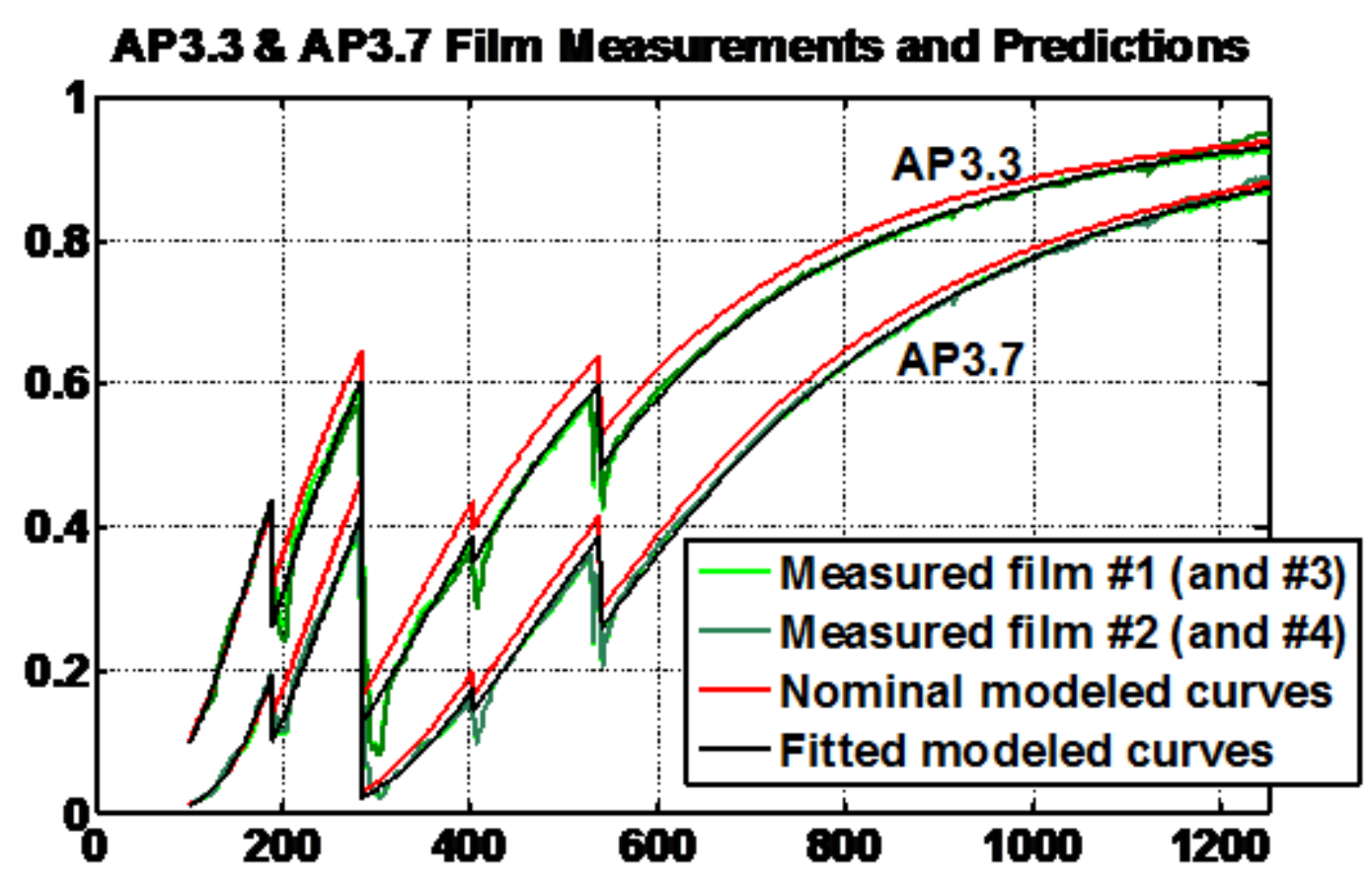

Figure 1. X-ray transmission of Moxtek AP3 windows at ALS compared to nominal fitted curves of Moxtek AP3 windows 